\title{
Les médecins suisses sont ils vraiment des «fat cats»?
}

\section{Alain de Weck}

Prof. méd. en retraite, Université de Berne
«La Suisse, il faut le dire aux médecins, est un des pays qui paient le mieux les médecins par rapport à l'ensemble de la population ... Le coefficient suisse relatif au rapport entre revenu des médecins et revenu moyen, est plus favorable aux médecins que dans n'importe quel autre pays parmi ceux qui nous entourent, et qu'aux Etats-Unis même où vous avez des écarts de revenus extraordinaires entre médecins. Or, en Suisse, on est d'accord de rémunérer les médecins bien mieux par rapport à la moyenne d'autres pays.»

Pascal Couchepin, Conseil des Etats 18.12.2008 [1].

Dans le cadre d'une étude approfondie du système de santé suisse [2], conduite non sous l'angle de l'ex-professeur de médecine mais sous celui du citoyen à la retraite qui a beaucoup de temps pour lire, je me suis appuyé les protocoles verbatim des séances du Conseil National et du Conseil des Etats de ces dernières années sur le sujet de la santé. Ces protocoles ne sont pas ennuyeux, comme d'aucuns pourraient le croire, mais se lisent en fait comme un roman. Ils font mieux comprendre les blocages sur le sujet depuis 2003. Au contraire des rapports de presse toujours édulcorés et des interviews, ils jettent une lumière crue et véridique sur l'intellect et le caractère de nos parlementaires et sur l'idéologie qui les anime. Certains passages, comme l'anicroche entre Anita Fetz et Bruno Frick [3] sont des morceaux d'anthologie. Je recommande vivement cette lecture avant les prochaines élections fédérales: elle est plus révélatrice sur les candidats que n'importe quel tract électoral ou programme de parti. minué en 40 ans de 40\% (revenu corrigé par inflation et/ou produit intérieur brut) alors que celui des ouvriers et employés a augmenté de 30\% durant la même période [4]. On peut bien se plaindre et entrer en grève pour une diminution injustifiée des tarifs de laboratoire ou l'abolition de la vente de médicaments par les médecins (propharmacie). Cela ne fait pleurer personne. Comment voulez vous que la grande majorité des Suisses qui gagnent 60000 francs par an ou moins, s'apitoient sur le sort de ceux qui en gagnent 200000 ou plus?

Cela d'autant plus lorsque la plus haute autorité du pays vient confirmer que les médecins suisses sont les mieux payés du monde et qu'une des solutions du contrôle des coûts consistera à leur faire rendre gorge. La presse quotidienne souffle dans la même trompette. Et les dirigeants de santésuisse n'y vont pas non plus avec le dos de la cuiller, continuant à affirmer publiquement [5] que «plusieurs milliers de médecins gagnent plus d'un million par an», un mensonge crasse (contre-vérité, en langue de bois) démenti par toutes les statistiques officielles.

Qu'en est il en réalité? D'après Pascal Couchepin [1], «si vous prenez le revenu moyen des Suisses et celui des médecins, et que vous divisez l'un par l'autre, vous obtenez un coefficient. Plus le coefficient est élevé, plus les médecins sont favorisés par rapport au revenu moyen».

C'est précisément la méthode utilisée par une étude récente de l'OCDE [6] qui compare les revenus des médecins dans 14 pays (USA, Canada, UK, Pays-Bas, Allemagne, Islande, Autriche, Luxembourg, France, Finlande, République tchèque, Danemark, Hongrie,

\section{Dans la conscience populaire les médecins sont des chats bien nourris, qui auraient bien tort de se plaindre}

Correspondance: Prof. Alain de Weck Beaumont 18 CH-1700 Fribourg alain.dewc@bluewin.ch
Dans le débat sur le système de santé, un thème reste central si ce n'est parfois sous-jacent: celui du revenu des médecins. Dans la conscience populaire, les médecins sont des «fats cats», des chats bien nourris, qui auraient bien tort de se plaindre. La FMH a beau publier les revenus annuels des médecins, basés sur des chiffres indiscutables (revenus AVS). Elle a beau démontrer que le pouvoir d'achat des médecins a di-
Suisse). Ironiquement, cette étude a été publiée le même jour que celui ou notre ministre de la santé proférait sa diatribe contre les médecins. Les résultats de cette étude, qui ne saurait être accusée de partialité, sont absolument clairs.

Selon l'OCDE et choisissant le critère de Pascal Couchepin, la rémunération des médecins par rapport au salaire moyen varie d'un pays à l'autre de 2,4 à 3,4 
pour les généralistes indépendants (fig. 1). La Suisse figure au $7^{\text {ème }}$ rang $(2,6)$, derrière les USA, l'Allemagne, le Canada, l'Autriche, la Grande-Bretagne et les PaysBas, mais au même niveau que la France. En termes de revenu réel, ajusté au pouvoir d'achat respectif, les généralistes suisses indépendants figurent également au $7^{\text {ème }}$ rang (108000 USD ajustés) derrière les USA (146000 USD), la Grande Bretagne (121000 USD), les Pays-Bas (120000 ISD), l'Allemagne (112000 USD)

Figure 1

Rémunérations des généralistes par rapport au salaire moyen dans 12 pays de I'OCDE (Source: OCDE: Health Working Report No 41, 2008).

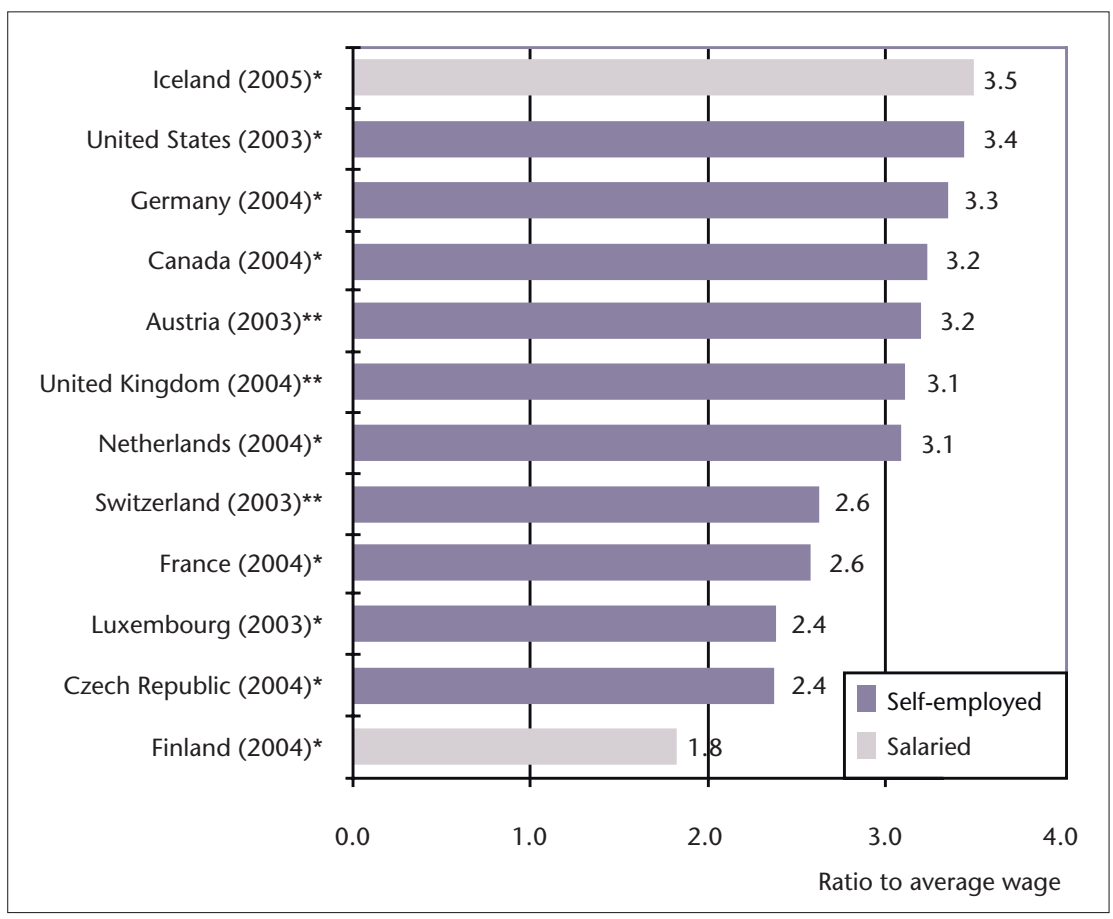

Figure 2

Rémunération des spécialistes par rapport au salaire moyen dans 13 pays de l'OCDE (Source: OCDE: Health Working Report No 41, 2008).

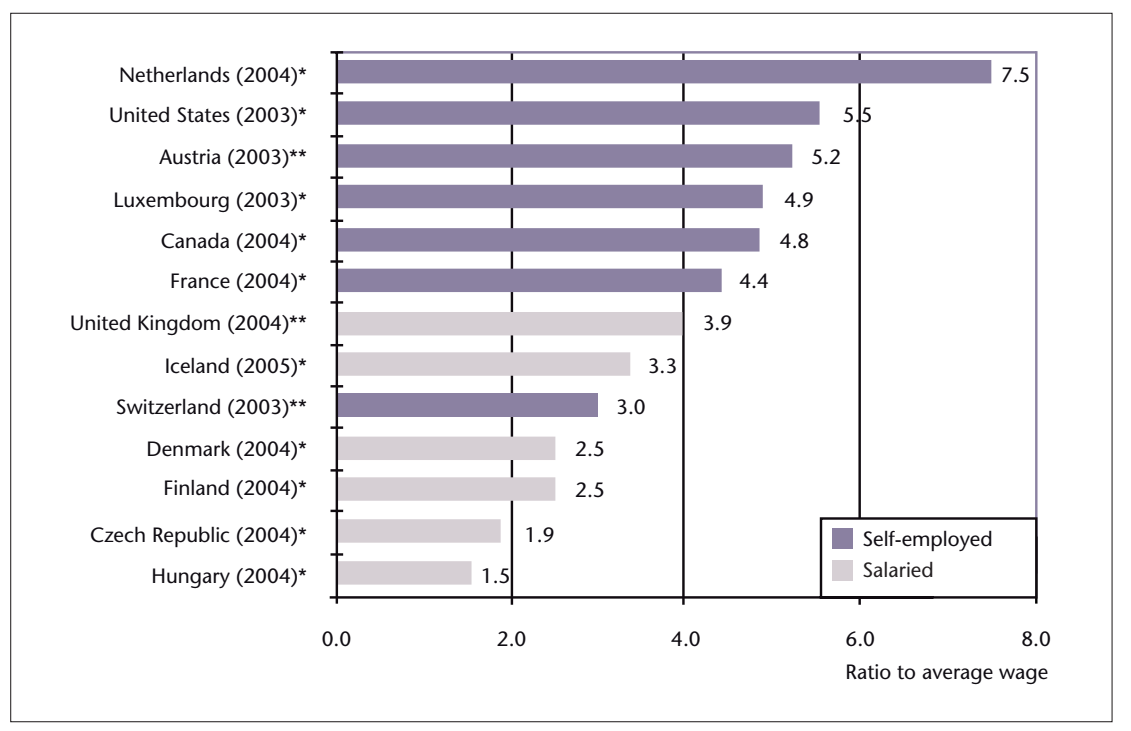

l'Autriche (108000 USD) et le Luxembourg (108000 USD).

Pour les spécialistes indépendants (fig. 2), les différences de rapport entre rémunération du médecin et salaire moyen sont nettement plus accentuées $(3,0-7,5)$. Dans cette comparaison, la Suisse ne figure qu'au $9^{\text {ème }}$ rang $(3,0)$ loin derrière les Pays-Bas $(7,5)$, les USA $(5,5), l^{\prime}$ Autriche $(5,2)$, le Luxembourg $(4,9)$ le Canada $(4,8)$ et la France $(4,4)$. En termes de pouvoir d'achat réel ajusté, l'échelle est la même: la Suisse (123000 USD) arrive loin derrière les Pays-Bas (290000 USD) et les USA (236000 USD).

Il est également intéressant de constater que la densité médicale n'a pas d'effet sur la rémunération des généralistes mais a un effet inverse sur la rémunération des spécialistes (fig. 3). Plus la densité de ces derniers est forte, plus leur revenu est faible, ce qui est au fond assez logique.

En ce qui concerne l'évolution du revenu des médecins et de leur pouvoir d'achat ces dernières 15 années, l'étude de l'OCDE fournit également des renseignements intéressants. Dans la plupart des pays, le revenu réel ajusté des généralistes a augmenté de 1995 à 2005 à des degrés variables mais généralement similaires à l'augmentation du salaire moyen (étude OCDE, fig 3). D'une manière générale, le revenu ajusté des médecins généralistes a augmenté par rapport au salaire moyen: Canada $(+7,6 \%)$, République tchèque $(+16,6 \%)$, Finlande $(+11,5 \%)$, France $(+12,1 \%)$, Allemagne $(+7,2 \%)$, Royaume Uni $(+43,7 \%)$. Il n'y a qu'en Autriche $(-4,3 \%)$ et aux USA (-6\%) que la rémunération des généralistes n'a pas augmenté.

\section{La Suisse semble être le seul des pays comparés par I'OCDE où le revenu ajusté des médecins par rapport au salaire moyen a diminué}

En ce qui concerne les spécialistes (OCDE, fig. 3), l'augmentation de la rémunération ajustée de 1995 à 2005 par rapport au salaire moyen est générale: Autriche $(+9 \%)$, Canada $(+7,6 \%)$, République tchèque $(+39,7 \%)$, Danemark $(+13,2 \%)$, Finlande $(+16,3 \%)$, France $(+26,4 \%)$, Royaume Uni $(+30,4 \%)$. Aux Etats Unis, la rémunération des spécialistes est restée stable $(-2,7 \%)$.

En fait, la Suisse semble être le seul des pays comparés par l'OCDE où le revenu ajusté des médecins par rapport au salaire moyen a diminué de 1995 à 2005. En effet, d'après les statistiques de la FMH [4], le pouvoir d'achat des médecins a diminué d'environ $5 \%$ durant cette période par rapport à l'indice des prix alors que le salaire moyen des ouvriers et employés augmentait d'environ 10,5\%, creusant donc l'écart comparatif à environ $15 \%$. 


\section{Figure 3}

Rémunération des généralistes et des spécialistes par rapport au salaire moyen et à la densité édicale dans 13 pays de I'OCDE (Source: OCDE: Health Working Report No 41, 2008).

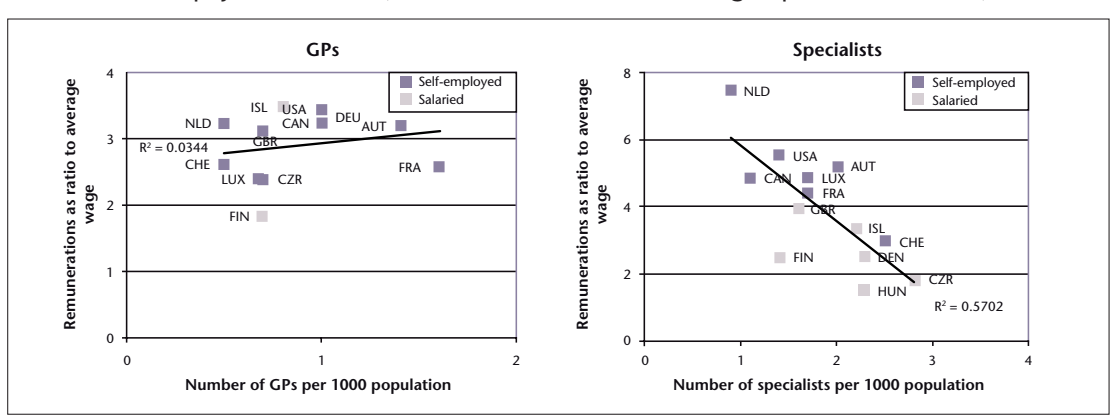

Certes, celui qui gagne 200000 francs par an peut difficilement prétendre à la compassion de celui qui en gagne 60000. Et beaucoup ne manquent pas de jouer sur cette fibre populiste. Il est inutile de rappeler que la médecine est un beau métier, que la plupart l'exercent pour d'autres motifs que des appétits pécuniaires et qu'il nécessite de longues études et beaucoup de sacrifices familiaux. Mais nos politiques devraient cesser de prétendre que les médecins suisses sont les plus grassement payés au monde. Ils devraient également cesser de considérer les médecins comme les cibles prioritaires des économies. Et certains dirigeants de santésuisse devraient cesser de clamer des contrevérités inqualifiables [5]. Dans l'intérêt d'un dialogue ouvert et rationnel qui reste indispensable entre les divers acteurs du système, il serait temps que l'on revienne aux réalités.

\section{Références}

1 Conseil des Etats, Séance du 18.12.2008. Révision LAMal. www.parlament.ch

2 De Weck A. Le système de santé suisse: un essai d'analyse citoyenne. 2010. En préparation.

3 Anita Fetz. 06.471 Initiative - Devoir de récusation pour les mandataires des caisses-maladie dans les affaires qui concernent l'assurance maladie sociale. 2008; www.parlament.ch

4 Hasler N, Reichert M. Revenus des médecins indépendants de Suisse en 2004 (réévaluation) et 2005 (nouveau). Bull Méd Suisses. 2009;90(11): 409-15.

5 Nelio Castelli. TSR Infrarouge. 6.10.2009. www.tsr.info.ch

6 OCDE Health Working Paper No 41. The remuneration of general practitioners and specialists in 14 OECD countries: What are the factors influencing variations across countries? 19.12.2008. ww.oecd.org/dataoecd/51/48/41925333.pdf

\section{Sie lesen gerade eine Zeitschrift des Schweizerischen Ärzteverlags EMH}

\section{Wussten Sie schon,}

dass EMH ein Gemeinschaftsunternehmen der Verbindung der Schweizer Ärztinnen und Ärzte FMH und der Schwabe AG ist, dem mit Gründung 1488 ältesten Druck- und Verlagshaus der Welt?

dass EMH mit insgesamt zehn Fachzeitschriften, einem umfangreichen Online-Angebot sowie einem wachsenden Buchprogramm der führende Verlag für medizinische Zeitschriften in der Schweiz ist?

- dass sämtliche bei EMH erscheinenden Zeitschriften offizielle Publikationsorgane der jeweils zuständigen medizinischen Fachorganisationen sind?

Wenn Sie mehr über EMH wissen möchten, finden Sie unter www.emh.ch weitere Informationen.

EMH Schweizerischer Ärzteverlag - Publikationen am Puls der Medizin 